\title{
A Density Result Concerning Inverse Polynomial Images*
}

\author{
Klaus Schiefermayr ${ }^{\dagger}$
}

\begin{abstract}
In this paper, we consider polynomials of degree $n$, for which the inverse image of $[-1,1]$ consists of two Jordan arcs. We prove that the four endpoints of these arcs form an $\mathcal{O}(1 / n)$-net in the complex plane.
\end{abstract}

Mathematics Subject Classification (2000): 30C10, 30E10, 33E05, 41A50

Keywords: Density result, Inverse polynomial image, Jacobian elliptic function, Jordan $\operatorname{arc}$

\section{Introduction and Main Result}

Let $\mathbb{P}_{n}$ be the set of all polynomials of degree $n$ with complex coefficients. For a polynomial $\mathcal{T}_{n} \in \mathbb{P}_{n}$, consider the inverse image of $[-1,1]$ defined by

$$
\mathcal{T}_{n}^{-1}([-1,1]):=\left\{z \in \mathbb{C}: \mathcal{T}_{n}(z) \in[-1,1]\right\}
$$

Inverse polynomial images are interesting for instance in approximation theory, since each polynomial $\mathcal{T}_{n} \in \mathbb{P}_{n}$ is (suitable normed) the Chebyshev polynomial, i.e. the minimal polynomial with respect to the supremum norm, of degree $n$ on its inverse image $\mathcal{T}_{n}^{-1}([-1,1])$, see [5], [7], [8], or [4].

In the following, we will need the notion of Jordan arcs. A set $\{\gamma(t) \in \overline{\mathbb{C}}: t \in[0,1]\}$ is called a Jordan arc if $\gamma:[0,1] \rightarrow \overline{\mathbb{C}}$ is continuous and $\gamma:[0,1) \rightarrow \overline{\mathbb{C}}$ is injective.

It is well known that $\mathcal{T}_{n}^{-1}([-1,1])$ is the union of $n$ Jordan arcs. The number of Jordan arcs can be reduced for some polynomials $\mathcal{T}_{n}$ : the inverse image $\mathcal{T}_{n}^{-1}([-1,1])$ consists of one Jordan arc if and only if $\mathcal{T}_{n}(z)=T_{n}(a z+b)$, where $T_{n}$ is the classical Chebyshev polynomial of the first kind, i.e. $T_{n}(z)=\cos (n \arccos z)$, see [12, Cor. 1]. In this case the inverse image is an interval in the complex plane (which of course can be seen as the union of $n$ intervals). In other words, the case of one Jordan arc is trivial.

In this note, we are interested in polynomials with an inverse image consisting of two Jordan arcs. Given four pairwise distinct points $a_{1}, a_{2}, a_{3}, a_{4} \in \mathbb{C}$ in the complex plane, define

$$
\mathcal{H}_{4}(z):=\left(z-a_{1}\right)\left(z-a_{2}\right)\left(z-a_{3}\right)\left(z-a_{4}\right) .
$$

Then the following characterization theorem holds, see [12, Thm. 1] or [10, Thm.3].

${ }^{*}$ will be published in: Proceedings of the AMS (2013).

${ }^{\dagger}$ University of Applied Sciences Upper Austria, School of Engineering and Environmental Sciences, Stelzhamerstr. 23, 4600 Wels, Austria, KLAus.SCHIEFERMAYR@FH-WELS.AT 
Theorem 1. Let $\mathcal{T}_{n}(z)=\tau z^{n}+\ldots \in \mathbb{P}_{n}$ be any polynomial of degree $n$. Then $\mathcal{T}_{n}^{-1}([-1,1])$ consists of two (but not less than two) Jordan arcs with endpoints $a_{1}, a_{2}, a_{3}, a_{4}$ if and only if $\mathcal{T}_{n}^{2}-1$ has exactly 4 pairwise distinct zeros $a_{1}, a_{2}, a_{3}, a_{4}$ of odd multiplicity, i.e., if and only if $\mathcal{T}_{n}$ satisfies a polynomial equation of the form

$$
\mathcal{T}_{n}^{2}(z)-1=\mathcal{H}_{4}(z) \mathcal{U}_{n-2}^{2}(z)
$$

with $\mathcal{U}_{n-2}(z)=\tau z^{n-2}+\ldots \in \mathbb{P}_{n-2}$ and $\mathcal{H}_{4}$ given in (2). In this case, the tuple $\left(a_{1}, a_{2}, a_{3}, a_{4}\right) \in \mathbb{C}^{4}$ is called a $\mathbf{T}_{n}$-tuple.

Condition (3) implies that $\mathcal{T}_{n}^{-1}([-1,1])$ consists of two Jordan arcs, which are not necessarily analytic. Concerning the minimum number of analytic Jordan arcs, we refer to [12, Thm. 3].

Now, we are able to state the main result. Roughly spoken it says that all $\mathbf{T}_{n}$-tuples $\left(a_{1}, a_{2}, a_{3}, a_{4}\right)$ form an $\mathcal{O}\left(\frac{1}{n}\right)$-net in the complex plane.

Theorem 2. Let $a_{1}, a_{2}, a_{3}, a_{4} \in \mathbb{C}$ be four paiwise distinct points in the complex plane. Then there exist $\tilde{a}_{2}, \tilde{a}_{3} \in \mathbb{C}$ such that $\left(a_{1}, \tilde{a}_{2}, \tilde{a}_{3}, a_{4}\right)$ is a $\mathbf{T}_{n}$-tuple and

$$
\left|a_{2}-\tilde{a}_{2}\right| \leq \frac{C_{1}}{n} \quad \text { and } \quad\left|a_{3}-\tilde{a}_{3}\right| \leq \frac{C_{2}}{n}
$$

holds for $n \geq N$, where $C_{1}, C_{2}, N$ depend only on $a_{1}, a_{2}, a_{3}, a_{4}$ but do not depend on $n$.

Remark. (i) The proof of Theorem 2 is given in the next section.

(ii) The values $C_{1}$ and $C_{2}$ can be expressed using Jacobian elliptic functions, see (26) and (27) in the proof of Theorem2, respectively.

(iii) For the real case, i.e. $a_{1}, a_{2}, a_{3}, a_{4} \in \mathbb{R}$, the density result of Theorem 2 has been proved long time ago by Achieser [1].

(iv) For the special case $a_{1}, a_{2} \in \mathbb{R}, a_{3} \in \mathbb{C} \backslash \mathbb{R}, a_{4}=\bar{a}_{3}$, the density result of Theorem2 is proved in [11.

(v) For the case of $\ell$ real intervals, a similar density result to that of Theorem 2 has been proved independently about the same time by Bogatyrev [2], Peherstorfer [9], and Totik [14].

\section{Proof of the Main Result}

The proof of the main result is managed with the help of the characterization of a $\mathbf{T}_{n}$-tuple using Jacobian elliptic and theta functions, see [10]. To this end, let us briefly recall some definitions. For an introduction to elliptic functions and integrals, we refer to [3] and [6].

Let $k \in D_{k}, D_{k}$ defined in (6) , be the modulus of the Jacobian elliptic functions $\operatorname{sn}(u)=\operatorname{sn}(u, k), \operatorname{cn}(u)=\operatorname{cn}(u, k)$, and $\operatorname{dn}(u)=\operatorname{dn}(u, k)$. Let $k^{\prime}$, defined by $k^{\prime 2}:=1-k^{2}$, be the complementary modulus and let $K=K(k)$ the complete elliptic integral of the first kind and let $K^{\prime}=K^{\prime}(k):=K\left(k^{\prime}\right)$.

Let $a_{1}, a_{2}, a_{3}, a_{4} \in \mathbb{C}$ be four pairwise distinct complex points and define the modulus $k$ by

$$
k^{2}:=\frac{\left(a_{4}-a_{1}\right)\left(a_{3}-a_{2}\right)}{\left(a_{4}-a_{2}\right)\left(a_{3}-a_{1}\right)} .
$$


By optionally exchanging some of the $a_{i}$ 's, it is always possible to get a tuple $\left(a_{1}, a_{2}, a_{3}, a_{4}\right)$ which satisfies

$$
\left|a_{4}-a_{1}\right| \cdot\left|a_{3}-a_{2}\right| \leq\left|a_{4}-a_{2}\right| \cdot\left|a_{3}-a_{1}\right|,
$$

i.e., $\left|k^{2}\right| \leq 1$, and for which $k^{2} \notin \mathbb{R}^{-}$. Obviously $k^{2}=0$ if and only if ( $\left.a_{1}=a_{4} \vee a_{2}=a_{3}\right)$ and $k^{2}=1$ if and only if $\left(a_{1}=a_{2} \vee a_{3}=a_{4}\right)$. In order to get $k$, we choose that branch of the square root in (4), for which $\operatorname{Re}(k)>0$. Therefore, we have to consider the case

$$
k \in D_{k}:=\{u \in \mathbb{C}:|u| \leq 1, \operatorname{Re}(u)>0, u \neq 1\}
$$

in the following. For $k \in D_{k}$, the functions $K=K(k)$ and $K^{\prime}=K^{\prime}(k)$ are single valued (for a detailed discussion see [6, Sect. 8.12]). For the complementary modulus $k^{\prime}$, by (4) and relation $k^{\prime 2}=1-k^{2}$, we get

$$
k^{2}=\frac{\left(a_{4}-a_{3}\right)\left(a_{2}-a_{1}\right)}{\left(a_{4}-a_{2}\right)\left(a_{3}-a_{1}\right)} .
$$

Further, we will need the function $\operatorname{sn}^{2}(u)$, which is an even elliptic function of order 2 with fundamental periods $2 K$ and $2 \mathrm{i} K^{\prime}$, with a double zero at 0 and a double pole at $\mathrm{i} K^{\prime}$. Further, for $k \in D_{k}$, let

$$
\mathcal{P}:=\left\{\mu K+\mathrm{i} \mu^{\prime} K^{\prime}: 0 \leq \mu, \mu^{\prime} \leq 1 \vee\left(0<\mu<1 \wedge-1<\mu^{\prime}<0\right)\right\}
$$

be a "half" period parallelogram of $\operatorname{sn}^{2}(u)$ with respect to the modulus $k$. Note that $\mathrm{i} K^{\prime} / K \notin \mathbb{R}$. By the above mentioned properties of $\operatorname{sn}^{2}(u)$, the mapping $\operatorname{sn}^{2}: \mathcal{P} \rightarrow \overline{\mathbb{C}}$, $u \mapsto \operatorname{sn}^{2}(u)$, is bijective, hence for given $a_{1}, a_{2}, a_{3}, a_{4} \in \mathbb{C}$, there exists a unique $\varrho=$ $\lambda K+\mathrm{i} \lambda^{\prime} K^{\prime} \in \mathcal{P}$ with $\lambda, \lambda^{\prime} \in \mathbb{R}$ such that

$$
\operatorname{sn}^{2}(\varrho)=\operatorname{sn}^{2}\left(\lambda K+\mathrm{i} \lambda^{\prime} K^{\prime}\right)=\frac{a_{4}-a_{2}}{a_{4}-a_{1}} .
$$

Note that $\varrho=0, K, K+\mathrm{i} K^{\prime}, \mathrm{i} K^{\prime}$ is equivalent to $\operatorname{sn}^{2}(\varrho)=0,1,1 / k^{2}, \infty$, respectively, and that $\left(a_{4}-a_{2}\right) /\left(a_{4}-a_{1}\right)=0,1,1 / k^{2}, \infty$ is posssible only if $a_{4}=a_{2}, a_{2}=a_{1}, a_{2}=a_{1}$, $a_{4}=a_{1}$, respectively. Thus, since $a_{1}, a_{2}, a_{3}, a_{4}$ are pairwise distinct, we have $\varrho \notin\{0, K, K+$ $\left.\mathrm{i} K^{\prime}, \mathrm{i} K^{\prime}\right\}$.

In the following characterization theorem [10, Thm. 7], a necessary and sufficient condition is given such that $\left(a_{1}, a_{2}, a_{3}, a_{4}\right)$ is a $\mathbf{T}_{n}$-tuple.

Theorem 3. Let $n \in \mathbb{N}$, let $a_{1}, a_{2}, a_{3}, a_{4} \in \mathbb{C}$ be pairwise distinct and satisfy (5), and let $k \in D_{k}$ and $\varrho \in \mathcal{P}$ be defined by (4) and (8), respectively. Then $\left(a_{1}, a_{2}, a_{3}, a_{4}\right)$ is a $\mathbf{T}_{n}$-tuple if and only if $\varrho$ is of the form

$$
\varrho=\frac{m}{n} K+\mathrm{i} \frac{m^{\prime}}{n} K^{\prime}, \quad \text { where } m, m^{\prime} \in \mathbb{Z} .
$$

In Fig.11, the set $\mathcal{P}$, defined in (8), and all points $\varrho \in \mathcal{P}$ of the form (10) (where $n=6$ was chosen) are illustrated.

If (10) holds, i.e. if $\left(a_{1}, a_{2}, a_{3}, a_{4}\right)$ is a $\mathbf{T}_{n}$-tuple, then the corresponding polynomials $\mathcal{T}_{n}(z), \mathcal{U}_{n-2}(z)$, and $\mathcal{H}_{4}(z)$ of Theorem1 can be represented with a certain conformal mapping and with the help of Jacobi's elliptic and theta functions, for details we refer to [10].

Before we start with the proof of Theorem 2, let us state an inequality for the elliptic sine function, which will be crucial for the proof. 


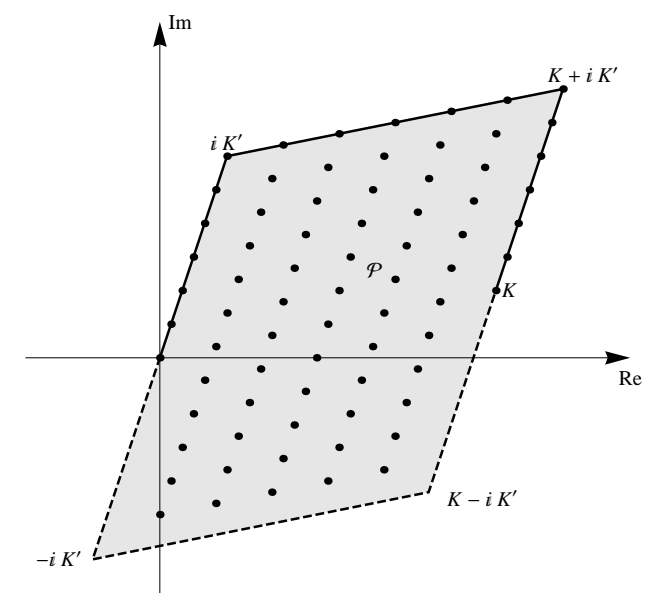

Figure 1: Illustration of the parallelogram $\mathcal{P}$

Lemma 1. Let $k \in D_{k}$, and $u \in \mathbb{C},|u| \leq \frac{\pi}{4}$. Then

$$
|\operatorname{sn}(u)| \leq \tan |u| \leq \frac{4}{\pi}|u| .
$$

Proof. A more general version of the first inequality is proved in [13]. The second inequality follows immediately from $f(x):=\tan x-\frac{4}{\pi} x \leq 0,0 \leq x \leq \frac{\pi}{4}$, since $f(0)=f\left(\frac{\pi}{4}\right)=0$ and $f^{\prime \prime}(x)=2 \sin x / \cos ^{3} x>0$ for $0 \leq x \leq \frac{\pi}{4}$.

Proof of Theorem[2. Let $n \in \mathbb{N}$ and let us assume that $a_{1}, a_{2}, a_{3}, a_{4}$ satisfy inequality (5) (which is always possible by a reordering of the $a_{j}$ ). Let $k$ be defined by (4), let $\varrho \in \mathcal{P}$ be defined by equation (9) and let $\lambda, \lambda^{\prime} \in \mathbb{R}$ be uniquely defined by

$$
\varrho=\lambda K+\mathrm{i} \lambda^{\prime} K^{\prime}
$$

By (4), (17), and (9),

$$
a_{2}=a_{4}-\left(a_{4}-a_{1}\right) \operatorname{sn}^{2}(\varrho)
$$

and

$$
a_{3}=\frac{a_{4}-k^{\prime 2} a_{1}}{k^{2}} \cdot \frac{a_{2}+\frac{k^{2} a_{1} a_{4}}{k^{\prime 2} a_{1}-a_{4}}}{a_{2}+\frac{k^{\prime 2} a_{4}-a_{1}}{k^{2}}}=A_{1} \cdot \frac{a_{2}+A_{2}}{a_{2}+A_{3}},
$$

where

$$
A_{1}:=\frac{a_{4}-k^{\prime 2} a_{1}}{k^{2}}, \quad A_{2}:=\frac{k^{2} a_{1} a_{4}}{k^{\prime 2} a_{1}-a_{4}}, \quad A_{3}:=\frac{k^{\prime 2} a_{4}-a_{1}}{k^{2}} .
$$

Clearly there exist integers $m, m^{\prime} \in \mathbb{Z}$ such that

$$
\left|\frac{m}{n}-\lambda\right| \leq \frac{1}{n} \quad \text { and } \quad\left|\frac{m^{\prime}}{n}-\lambda^{\prime}\right| \leq \frac{1}{n} .
$$

Let us remark that the integers $m, m^{\prime}$ can be chosen such that (cf. Fig. 1)

$$
\varrho \pm \tilde{\varrho} \notin\left\{\nu K+\mathrm{i} \nu^{\prime} K^{\prime}: \nu, \nu^{\prime} \in \mathbb{Z}\right\},
$$


where $\tilde{\varrho}$ is defined by

$$
\tilde{\varrho}:=\frac{m}{n} K+\mathrm{i} \frac{m^{\prime}}{n} K^{\prime} .
$$

Note that (16) implies that none of the points $\varrho \pm \tilde{\varrho}$ or $\frac{1}{2}(\varrho \pm \tilde{\varrho})$ is a pole of $\operatorname{sn}(u), \operatorname{cn}(u)$, or $\operatorname{dn}(u)$. Define

$$
\tilde{a}_{2}:=a_{4}-\left(a_{4}-a_{1}\right) \operatorname{sn}^{2}(\tilde{\varrho})
$$

and

$$
\tilde{a}_{3}:=\frac{a_{4}-k^{\prime 2} a_{1}}{k^{2}} \cdot \frac{\tilde{a}_{2}+\frac{k^{2} a_{1} a_{4}}{k^{\prime 2} a_{1}-a_{4}}}{\tilde{a}_{2}+\frac{k^{\prime 2} a_{4}-a_{1}}{k^{2}}}=A_{1} \cdot \frac{\tilde{a}_{2}+A_{2}}{\tilde{a}_{2}+A_{3}},
$$

where the last equality follows from (14). Then

$$
k^{2}=\frac{\left(a_{4}-a_{1}\right)\left(\tilde{a}_{3}-\tilde{a}_{2}\right)}{\left(a_{4}-\tilde{a}_{2}\right)\left(\tilde{a}_{3}-a_{1}\right)}
$$

and

$$
\operatorname{sn}^{2}(\tilde{\varrho})=\frac{a_{4}-\tilde{a}_{2}}{a_{4}-a_{1}} .
$$

holds. Note that the modulus $k$ for the tuples $\left(a_{1}, a_{2}, a_{3}, a_{4}\right)$ and $\left(a_{1}, \tilde{a}_{2}, \tilde{a}_{3}, a_{4}\right)$ is the same. By (17), (20), (21) and Theorem3, $\left(a_{1}, \tilde{a}_{2}, \tilde{a}_{3}, a_{4}\right)$ is a $\mathbf{T}_{n}$-tuple. By (12) and (18),

$$
\begin{aligned}
& \left|a_{2}-\tilde{a}_{2}\right|=\left|a_{4}-a_{1}\right| \cdot\left|\operatorname{sn}^{2}(\varrho)-\operatorname{sn}^{2}(\tilde{\varrho})\right| \\
& \quad=\left|a_{4}-a_{1}\right| \cdot|\operatorname{sn}(\varrho)-\operatorname{sn}(\varrho)| \cdot|\operatorname{sn}(\varrho)+\operatorname{sn}(\tilde{\varrho})| \\
& \quad=\left|a_{4}-a_{1}\right| \cdot|\operatorname{sn}(\varrho)+\operatorname{sn}(\varrho)| \cdot \frac{\left|2 \operatorname{sn}\left(\frac{1}{2} \varrho-\frac{1}{2} \varrho\right) \operatorname{cn}\left(\frac{1}{2} \varrho+\frac{1}{2} \varrho\right) \operatorname{dn}\left(\frac{1}{2} \varrho+\frac{1}{2} \varrho\right)\right|}{\left|1-k^{2} \operatorname{sn}^{2}\left(\frac{1}{2} \varrho+\frac{1}{2} \tilde{\varrho}\right) \operatorname{sn}^{2}\left(\frac{1}{2} \varrho-\frac{1}{2} \varrho\right)\right|}
\end{aligned}
$$

where in the last equation the well-known formula for $\operatorname{sn}(u)-\operatorname{sn}(v)$ is used, see, e.g., [3, (123.06)]. By (11), (15), and (17),

$$
\begin{aligned}
|\varrho-\tilde{\varrho}| & =\left|\left(\lambda-\frac{m}{n}\right) K+\mathrm{i}\left(\lambda^{\prime}-\frac{m^{\prime}}{n}\right) K^{\prime}\right| \\
& \leq\left|\lambda-\frac{m}{n}\right| \cdot|K|+\left|\lambda^{\prime}-\frac{m^{\prime}}{n}\right| \cdot\left|K^{\prime}\right| \\
& \leq \frac{|K|+\left|K^{\prime}\right|}{n} .
\end{aligned}
$$

If

$$
n \geq \frac{2}{\pi}\left(|K|+\left|K^{\prime}\right|\right)=: n_{1}
$$

then, by (22),

$$
\left|\frac{1}{2} \varrho-\frac{1}{2} \tilde{\varrho}\right| \leq \frac{\pi}{4}
$$

Thus, using Lemma 1 and (22),

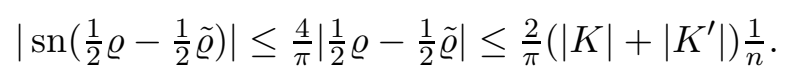

Summing up, for $n \geq n_{1}$, we have the inequality

$$
\left|a_{2}-\tilde{a}_{2}\right| \leq \frac{2}{n \pi}\left(|K|+\left|K^{\prime}\right|\right)\left|a_{4}-a_{1}\right| B .
$$


where

$$
B:=|\operatorname{sn}(\varrho)+\operatorname{sn}(\tilde{\varrho})| \cdot \frac{\left|2 \operatorname{cn}\left(\frac{1}{2} \varrho+\frac{1}{2} \tilde{\varrho}\right) \operatorname{dn}\left(\frac{1}{2} \varrho+\frac{1}{2} \tilde{\varrho}\right)\right|}{\left|1-k^{2} \operatorname{sn}^{2}\left(\frac{1}{2} \varrho+\frac{1}{2} \varrho\right) \operatorname{sn}^{2}\left(\frac{1}{2} \varrho-\frac{1}{2} \tilde{\varrho}\right)\right|}
$$

Since $\varrho \notin\left\{0, K, \pm \mathrm{i} K^{\prime}, K \pm \mathrm{i} K^{\prime}\right\}$, there exists an $n_{2} \in \mathbb{N}$ such that $0, K, \pm \mathrm{i} K^{\prime}, K \pm \mathrm{i} K^{\prime} \notin$ $\mathcal{P}\left(n_{2}\right)$, where

$$
\mathcal{P}\left(n_{2}\right):=\left\{u \in \mathbb{C}: u=\mu K+\mathrm{i} \mu^{\prime} K^{\prime},|\mu-\lambda| \leq \frac{1}{n_{2}},\left|\mu^{\prime}-\lambda^{\prime}\right| \leq \frac{1}{n_{2}}, \mu, \mu^{\prime} \in \mathbb{R}\right\} .
$$

Thus, the maxima

$$
s^{*}:=\max _{u \in \mathcal{P}\left(n_{2}\right)}|\operatorname{sn}(u)|, \quad c^{*}:=\max _{u \in \mathcal{P}\left(n_{2}\right)}|\operatorname{cn}(u)|, \quad d^{*}:=\max _{u \in \mathcal{P}\left(n_{2}\right)}|\operatorname{dn}(u)|
$$

exist. By construction of $\mathcal{P}\left(n_{2}\right)$, for $n \geq n_{2}$, we have $\varrho, \tilde{\varrho}, \frac{1}{2}(\varrho+\tilde{\varrho}) \in \mathcal{P}\left(n_{2}\right)$. Further, we have

$$
\left|k^{2}\right| \cdot\left|\operatorname{sn}^{2}\left(\frac{1}{2} \varrho+\frac{1}{2} \tilde{\varrho}\right)\right| \cdot\left|\operatorname{sn}^{2}\left(\frac{1}{2} \varrho-\frac{1}{2} \tilde{\varrho}\right)\right| \leq\left(s^{*}\right)^{2} \cdot \frac{4}{\pi^{2}}\left(|K|+\left|K^{\prime}\right|\right)^{2} \cdot \frac{1}{n^{2}} \leq \frac{1}{2},
$$

where the last inequality is true if

$$
n \geq n_{3}:=\frac{2 \sqrt{2} s^{*}}{\pi}\left(|K|+\left|K^{\prime}\right|\right) .
$$

Hence, for $B$ defined in (25),

$$
B \leq \frac{4 s^{*} c^{*} d^{*}}{|1-| k^{2}|\cdot| \operatorname{sn}^{2}\left(\frac{1}{2} \varrho+\frac{1}{2} \varrho\right)|\cdot| \operatorname{sn}^{2}\left(\frac{1}{2} \varrho-\frac{1}{2} \varrho\right)||} \leq 8 s^{*} c^{*} d^{*}
$$

and altogether, for $n \geq \max \left\{n_{1}, n_{2}, n_{3}\right\}$, we get the inequality

$$
\left|a_{2}-\tilde{a}_{2}\right| \leq \frac{C_{1}}{n}
$$

where

$$
C_{1}:=\frac{16}{\pi}\left(|K|+\left|K^{\prime}\right|\right)\left|a_{4}-a_{1}\right| s^{*} c^{*} d^{*}
$$

Using (13) and (19), we get

$$
\left|a_{3}-\tilde{a}_{3}\right|=\left|A_{1}\right| \cdot \frac{\left|a_{2}-\tilde{a}_{2}\right| \cdot\left|A_{2}-A_{3}\right|}{\left|a_{2}+A_{3}\right| \cdot\left|\tilde{a}_{2}+A_{3}\right|}
$$

and

$$
\left|\tilde{a}_{2}+A_{3}\right|=\left|a_{4}-a_{1}\right| \cdot\left|\operatorname{sn}^{2}(\tilde{\varrho})-1 / k^{2}\right| .
$$

Since $u=K+\mathrm{i} K^{\prime} \in \mathcal{P}$ is the only point in $\mathcal{P}$, for which $\operatorname{sn}^{2}(u)=1 / k^{2}$, by construction of $\mathcal{P}\left(n_{2}\right)$,

$$
s^{* *}:=\min _{u \in \mathcal{P}\left(n_{2}\right)}\left|\operatorname{sn}^{2}(u)-1 / k^{2}\right|>0
$$

holds. Thus

$$
\left|a_{3}-\tilde{a}_{3}\right| \leq \frac{C_{2}}{n}
$$

where

$$
C_{2}:=\frac{C_{1} \cdot\left|A_{1}\right| \cdot\left|A_{2}-A_{3}\right|}{\left|a_{2}+A_{3}\right| \cdot\left|a_{4}-a_{1}\right| \cdot s^{* *}}
$$

and $A_{1}, A_{2}, A_{3}$ are defined in (14). 


\section{References}

[1] N.I. Achieser, Über einige Funktionen, welche in zwei gegebenen Intervallen am wenigsten von Null abweichen, Bull. Acad. Sci. URSS 7 (1932), 1163-1202 (in German).

[2] A.B. Bogatyrëv, On the efficient computation of Chebyshev polynomials for several intervals, Sb. Math. 190 (1999), 1571-1605.

[3] P.F. Byrd and M.D. Friedman, Handbook of elliptic integrals for engineers and scientists, Springer, 1971.

[4] B. Fischer and F. Peherstorfer, Chebyshev approximation via polynomial mappings and the convergence behaviour of Krylov subspace methods, Electron. Trans. Numer. Anal. 12 (2001), 205-215 (electronic).

[5] S.O. Kamo and P.A. Borodin, Chebyshev polynomials for Julia sets, Moscow Univ. Math. Bull. 49 (1994), 44-45.

[6] D.F. Lawden, Elliptic functions and applications, Springer, 1989.

[7] I.V. Ostrovskii, F.B. Pakovitch, and M.G. Zaidenberg, A remark on complex polynomials of least deviation, Internat. Math. Res. Notices (1996), no. 14, 699-703.

[8] F. Peherstorfer, Minimal polynomials for compact sets of the complex plane, Constr. Approx. 12 (1996), 481-488.

[9] _ Deformation of minimal polynomials and approximation of several intervals by an inverse polynomial mapping, J. Approx. Theory 111 (2001), 180-195.

[10] F. Peherstorfer and K. Schiefermayr, Description of inverse polynomial images which consist of two Jordan arcs with the help of Jacobi's elliptic functions, Comput. Methods Funct. Theory 4 (2004), 355-390.

[11] K. Schiefermayr, Inverse polynomial images consisting of an interval and an arc, Comput. Methods Funct. Theory 9 (2009), 407-420.

[12] _ Geometric properties of inverse polynomial images, Proceedings Approximation Theory XIII: San Antonio 2010, Springer Proceedings in Mathematics 13, 2012, pp. $277-287$.

[13] Inequalities for the Jacobian elliptic functions with complex modulus, J. Math. Inequal. 6 (2012), 91-94.

[14] V. Totik, Polynomial inverse images and polynomial inequalities, Acta Math. 187 (2001), 139-160. 